\title{
Mitteilungen / Communications
}

Le Docteur Fred Paccaud. PD et agrégé, chef de la division des services de santé de l'Institut universitaire de médecine sociale et préventive de Lausanne, a été nommé Directeur de cet institut et Professeur ordinaire de médecine sociale et préventive à la Faculté de médecine de Lausanne. Il remplace dans ces fonctions le Professeur Felix Gutzwiller, qui a été nommé, en automne 1987. Directeur de l'Institut de médecine sociale et préventive de l'Université de Zu. rich et Professeur ordinaire de médecine sociale et préventive à la Faculté de médecine de Zurich.
«Salvatore Maugeri» Prize for research on «Biological Monitoring Methods of Carcinogenic Risk in Industry» Participants who wish to have their research considered for the Prize mentioned above will have to submit their documentation by December 31st 1988. Information: Fondazione Clinica Del Lavoro. Via P. Azzario. 19, I-27100 Pavia. Te]. 0382/37201.

\section{Balint - Preis für Medizinstudenten}

Monte Verità, Ascona

zur Förderung beziehungsorientierter Aus bildung. Einsendeschluss: 31.Dezember
1988. Auskunft: Balint-Dokumentationszentrum, $\mathrm{CH}-6612$ Ascona.

\section{Formation en Santé Publique} et Santé Communautaire

Université de Nancy I. Faculté de Médecine, Département de Santé Publique.

Formation continue. enseignement universitaire de $3^{\circ}$ cycle, 1987 - 1988. Information: B.P. 184. 54505 Vandœuvre-lès-Nancy Cedex. Tél. 83565656 .

\section{Voranzeigen / Dates à retenir}

\section{Internationales Balint-Treffen -} Ascona-Gespräche

31. März - 2. April 1989. Thema: Der alternde Mensch und sein Arzt. Auskunft Balint-Dokumentationszentrum 、 $\mathrm{CH}-6612$ Ascona

\section{First International Congress} on Peer Review in Biomedical Publication 10th to 12th May 1989 in Chicago. Informa tion: Ms. Sharon Iverson, Journal of the
American Medical Association, 535 North Dearborn Street, Chicago, Illinois 60610. (312) 280-7123

Pro Aqua-Pro Vita 89 - 11. Fachmesse für Umwelttechnik und Umweltschutz 6. - 9. Juni 1989 in den Hallen der Schweizer Mustermesse in Basel. Auskunft: Pro Aqua Pro Vita 89. Postfach. CH-4021 Basel. Tel. 0616862020 2nd International Conference on Preventive Cardiology

18th - 22th June 1989 in Washington. D.C. Deadline for abstracts December 15 th. 1988. Organized by the International Society and Federation of Cardiology. Secretary: Dr. A. Hofman. Dept. of Epidemiology. Faculty of Medicine. P.O. Box 1738. Erasmus University, 3000DR Rotterdam. The Netherlands

\section{Neue Bücher / Nouveaux livres}

\author{
Präventive Medizin \\ Aspekte und Perspektiven \\ einer vorbeugenden Medizin \\ H. Schaefer, H. Schipperges, G. Wagner \\ (Hrsg.), 307 S., 26. Abb., 17. Tab., \\ broschiert, Springer-Verlag Berlin, \\ Heidelberg, New York, 1987. \\ ISBN 3-540-17523-7
}

Das vorliegende Buch sammelt von $15 \mathrm{Au}$ toren Referate, die in den Jahren 1983 bis 1986 an einem interdisziplinären Kolloquium am Institut für Geschichte in der Medizin in Heidelberg unter dem Thema «Probleme einer präventiven Medizin" gehalten worden sind. Besonders lesenswert scheinen die Beiträge von Schäfer, Verres, Münnich und Wagner zu sein, auf die kurz eingegangen werden soll: Schäfer betrachtet die Problematik und die Chancen der Prävention und kommt zum Schluss, dass die Chancen gross seien, soweit es die sachlichen Argumente angehe, aber bescheidener, soweit es die Psychologie der Massen betreffe. Prävention ist eine Methode, die demjenigen, der ein Leben möglichst ohne Krankheiten wünscht, helfen kann. Sie sei aber in ihrer Massenwirkung weniger erfolgreich und spiegle die in der Gesellschaft herrschende Genusssucht. Verres schildert die psychologischen Voraussetzungen präventiven Verhaltens insbesondere der sozialen Unterschicht, bei der bekanntlich die Nichtteilnahme an den Vorsorgeuntersuchungen grösser ist und die sich durch folgende psychologische Merkmale auszeichnet: soziale Distanz zu Institutionen der Medizin, kognitive und sprachliche Differenzen, niedrigerer Wissensstand über Beeinflussbarkeit von Gesundheit und Krankheit, tendenziell eher kurzfristig orientierte Lebensplanung usw. Münnich geht der Frage nach, ob Prävention zur Kostendämpfung im Gesundheitswesen beitrage und schliesst daraus, dass Prävention im Einzelfall zwar kostensenkend wirken könne (Beispiel: Fluoridierung des Trinkwassers und Polio-Impfung), nicht aber als generell einsetzbares Mittel. Die Argumentation dafür lautet abgekürzt: Krank wird man immer, mit Hilfe der Prävention erkrankt man im höheren Alter als sonst. Die geriatrischen Krankheiten sind aber besonders teuer, da sie meistens chronisch sind. Wagner gibt schliesslich einen guten Überblick über den derzeitigen Stand der Prävention bei den Tumoren.

Zusammenfassend kann gesagt werden. dass das Buch von ganz verschiedenen Standpunkten den momentanen Entwicklungsstand der Prävention belichtet, auf ei- 